\title{
Perspectiva de profesionales de salud sobre la violencia de la pareja en el embarazo*
}

\author{
Perspective of the Health Professionals on the Partner Violence during Pregnancy \\ Perspectiva dos profissionais da saúde sobre a violência do casal na gravidez
}

\author{
Maria Mercedes Lafaurie Villamil ${ }^{a}$ \\ Universidad El Bosque, Colombia \\ lafauriemaria@unbosque.edu.co \\ ORCID: http://orcid.org/0000-0002-5993-740X \\ María Luisa Ramírez Romero ${ }^{1 .}$ \\ ORCID: http://orcid.org/0000-0002-2497-4956
}

DOI: https://doi.org/10.11144/Javeriana.ie21-2.ppsv

\section{Resumen:}

Introducción: la violencia de pareja (VP) durante el embarazo afecta los derechos de las gestantes y causa serias complicaciones para la madre o su hijo por nacer. El personal de salud puede enfrentar esta realidad mediante un adecuado proceso de detección y atención. Objetivo: explorar la perspectiva de profesionales de salud de la Unidad de Servicios de Salud Usaquén, de Bogotá, sobre la VP en el embarazo. Método: estudio cualitativo, entre marzo y diciembre de 2016. Se realizaron cuatro grupos focales con la participación de 38 profesionales. El análisis se desarrolló con apoyo del software ATLAS.ti 7. Resultados: la corta edad y la carencia de recursos y redes de apoyo perpetúa la VP. Los participantes sugieren la intervención de equipos multidisciplinarios coordinados en la detección y atención biopsicosocial de la problemática, construir ambientes de contención emocional a las usuarias, empoderarlas para el ejercicio de derechos y capacitar al personal de salud en el manejo de la problemática. Discusión: para el personal de salud es imperioso saber enfrentar esta realidad, de manera que las mujeres salgan fortalecidas. La inclusión de una mirada de género y derechos es una necesidad a la hora de abordar la VP en los contextos de la salud. Conclusiones: para un cambio de condiciones se requiere: a) trabajo multidisciplinario coordinado, b) ambientes amigables, c) atención humanizada a la gestante, d) reducción de la revictimización y e) capacitación al personal de salud para la intervención en VP.

Palabras clave: violencia contra la mujer, violencia de pareja, violencia doméstica, embarazo, investigación cualitativa, personal de salud.

\section{Abstract:}

Introduction: Intimate partner violence (IPV) during pregnancy undermines the pregnant woman's rights and brings about serious complications both to the mother and the unborn child. Health professionals will have to cope with this reality through an appropriate process of detection and care. Objective: To explore the perspective by the health professionals in the health center 'Unidad de Servicios de Salud Usaquén' in Bogotá, regarding the IPV during pregnancy. Method: A qualitative study was conducted between March and December 2016. Four focus groups were carried out with 38 health professional taking part in them. The analysis was developed by using the software ATLASti 7. Results: Being young, financial shortfalls and the lack of support feed into the IPV. The participants propose the intervention by coordinated multidisciplinary teams for the detection and bio-psychosocial care to people with this problem, to build emotional containment environments for the users, to empower the women so that they enforce their rights, and to train the health professionals for managing this problem. Discussion: It is a must that the health professionals become able to cope with this reality, so that they can strengthen the women in this situation. There is a need to provide them with a gender-oriented view and rights awareness when it comes to face the IPV in the contexts of health issues. Conclusions: To do a change in the conditions requires: $a$ ) coordinated multidisciplinary work; $b$ ) friendly environments; c) humanized care to pregnant women; d) to reduce the re-victimization; and e) to train the health workers in IPV intervention. Keywords: violence against women, intimate partner violence, domestic violence, pregnancy, qualitative research, health workers.

Notas de autor

\footnotetext{
a Autora de correspondencia. Correo electrónico: lafauriemaria@unbosque.edu.co

1. Consultora independiente en servicios de salud
} 


\section{Resumo:}

Introdução: A violência de casal (VC) durante a gravidez afeta os direitos das gestantes e ocasiona complicações fortes para mãe e filho ainda não nascido. O pessoal de saúde pode enfrentar essa realidade mediante um adequado processo de triagem e atendimento. Objetivo: Explorar a perspectiva dos profissionais da saúde da Unidade de Serviços de Saúde Usaquén, de Bogotá, sobre a VC na gravidez. Método: foi realizado um estudo qualitativo entre marco e dezembro de 2016. Foram realizados quatro grupos focais com participação de 38 profissionais. A análise desenvolveu-se com suporte do software ATLASti 7. Resultados: A pouca idade e a carência de recursos e redes de apoio perpetuam a VC. Os participantes sugerem a intervenção de equipes multidisciplinares coordenadas na detecção e atendimento biopsicossocial da problemática, construir ambientes de contenção emocional às usuárias, empoeirá-las para o exercício de direitos e treinar o pessoal de saúde na gestão da problemática. Discussão: Para o pessoal de saúde é imperativo saber enfrentar essa realidade, de jeito tal que as mulheres saírem fortalecidas. A inclusão de um olhar de gênero e direitos é uma necessidade à hora de abordar a VC nos contextos da saúde. Conclusões: Para uma mudança de condições é preciso: a) trabalho multidisciplinar coordenado b) ambientes amigáveis c) atendimento humanizado à gestante, d) redução da revitimização e e) treinamento ao pessoal de saúde para a intervenção em VC.

Palavras-chave: violência contra as mulheres, violência de casal, violência doméstica, gravidez, pesquisa qualitativa, pessoal de saúde.

\section{Introducción}

La violencia contra las mujeres es un problema de salud pública, con implicaciones en sus derechos humanos, que causa serias complicaciones en la salud mental y en la sexual y reproductiva (1). En el mundo, alrededor del $35 \%$ de las mujeres ha experimentado violencia física o sexual en algún momento de sus vidas, bien sea por parte de sus parejas o de otras personas (2).

Una de las formas más frecuentes de la violencia de género a las mujeres es la violencia de pareja (VP), que consiste en actos generados por la pareja o por la expareja "que causan daño físico o emocional, que pueden incluir coerción sexual o violación, intimidación física, intentos de muerte o daño, restricción de las actividades normales o de la libertad y negación de acceso a los recursos" (3). En Colombia y en otros países de América Latina es de cierta forma reciente el estudio y el reconocimiento de la violencia que se presenta contra las mujeres durante el embarazo (4). Las normas sociales y culturales parecen respaldar este tipo de violencia en la región de América Latina (5). En Colombia se observa una relación entre maltrato de la pareja y embarazo no deseado (6). De igual modo, en Perú se han hallado efectos de la violencia de la pareja íntima en la presentación de síntomas de depresión (7).

La VP tiene implicaciones negativas en el embarazo como sangrado vaginal, trauma abdominal, hemorragia, restricción del crecimiento intrauterino y muerte perinatal (8); de igual modo, en el recién nacido hay bajo peso al nacer (9) y depresión posparto en la mujer puérpera (8).

Las mujeres víctimas de violencia suelen solicitar más servicios de salud que otras mujeres (10); por ende, tanto la VP como la violencia sexual a las mujeres requieren ser abordadas científicamente con el fin de orientar acciones en torno a su prevención (11). De ahí que sea crucial el papel del equipo de salud para evitar su revictimización (12). Los profesionales de salud precisan hacer remisiones adecuadas para una atención de calidad a las gestantes que viven VP (5). El sector de la salud tiene la responsabilidad no solo de responder a la violencia subyacente que experimentan las mujeres que buscan servicios de salud, sino también de reconocer los factores contextuales implicados. Se debe empoderar a trabajadores sanitarios, mujeres y comunidades, así como eliminar barreras del sistema que impiden la atención de calidad. Una respuesta integral del sector de la salud a la VP tiene el potencial de mejorar esta situación (13).

En la localidad Usaquén, ubicada en la zona nororiental de Bogotá, se encuentra la Unidad de Servicios de Salud Usaquén (Antiguo Hospital de Usaquén), entidad de primer nivel comprometida con la erradicación de la violencia de género (14). Según la Primera Encuesta Distrital de Demografía y Salud, en Usaquén, un $72 \%$ de las mujeres afirma que han sido controladas por sus parejas; mientras que el $27 \%$ ha sido agredida físicamente (15). En zonas deprimidas de la localidad se ha informado violencia intrafamiliar que motiva la temprana salida del hogar y el embarazo en las adolescentes (16). En un reciente estudio con gestantes 
atendidas por esta unidad se hallaron narrativas sobre violencia de pareja (17). En concordancia con esa realidad, la entidad de salud consideró crucial conocer la visión del equipo de profesionales de salud. Por ello, el objetivo del estudio fue reconocer la perspectiva de miembros del equipo de salud institucional sobre la VP en el embarazo y sobre su detección y atención, con miras a contar con referentes para la construcción de lineamientos para la acción.

Dada la intencionalidad del estudio, el enfoque cualitativo resulta ser el más pertinente, por cuanto permite comprender una realidad desde el lugar de los actores implicados y obtener información relevante para suscitar el cambio, cuando aspectos como los contextos culturales y sociales se ven implicados. A pesar de su relevancia en el enfrentamiento de la problemática, en Colombia existe escasa información en la literatura científica sobre la mirada del personal de salud al problema y a sus posibles soluciones, por lo que los resultados de este trabajo pueden ser de interés para investigadores, profesionales y tomadores de decisiones en el campo del género y la salud de las mujeres.

\section{Método}

El estudio fue de tipo cualitativo, basado en la técnica de grupos focales. La entrevista de grupo focal es abierta y grupal; facilita el diálogo y la generación de un clima de confianza entre los participantes; fortalece la confiabilidad de los datos, al disminuir sesgos de opinión, y está especialmente indicada para tratar temas de salud (18), razón por la cual se seleccionó para este estudio. Los grupos focales están orientados por una epistemología cualitativa que se inscribe dentro de los modelos constructivo-interpretativos, los cuales les dan valor especial a las subjetividades y entienden el conocimiento como una construcción humana que emana a partir de procesos fundamentados en el diálogo y la comunicación (19). La investigación cualitativa facilita la práctica sensible basada en las necesidades de los pacientes (20).

El muestreo o proceso de selección de participantes para este estudio fue de tipo propositivo, de colección completa, que incluye, según Martínez-Salgado (21), todos aquellos sujetos que cumplan los criterios de selección que, en este caso, fueron los siguientes: ser profesionales de la salud, laborar en la Unidad de Servicios de Salud Usaquén y tener una experiencia mínima de seis meses de trabajo en esta. La selección de sujetos para grupos focales es intencional y se basa en los objetivos del estudio; reúne sujetos que comparten rasgos comunes (18).

Se convocaron a participar todos los profesionales de la Unidad de Servicios de Salud con más de seis meses de experiencia laboral en la entidad, partiendo de que todas las áreas de la Unidad (que es de primer nivel de atención) tienen la responsabilidad de trabajar para erradicar la violencia de género, están en contacto permanente con gestantes y pueden apoyar en la detección de la VP, así como aportar a su enfrentamiento. Participaron de manera voluntaria 38 profesionales de la salud de diferentes áreas, invitados a vincularse al proceso mediante una convocatoria escrita, quienes constituyeron cuatro grupos focales: catorce enfermeras, diez médicos y médicas, cuatro profesionales de trabajo social, seis psicólogos y psicólogas y cuatro profesionales de otras profesiones de salud (odontología, fonoaudiología y fisioterapia).

Las entrevistas de grupos focales, con cinco preguntas iniciales, se construyeron con base en los objetivos del estudio y fueron revisadas en su contenido por dos expertos. Para ello se usó un cuestionario de tipo cualitativo orientado por los objetivos del estudio, lo que dio pie a ajustes en la versión definitiva. Las preguntas buscaron suscitar reflexión y discusión sobre las formas de VP más frecuentes entre las gestantes usuarias de los servicios de la Unidad; los posibles antecedentes de esta situación; las estrategias sugeridas para la detección de la VP entre las usuarias embarazadas; las estrategias de atención que pueden tener mayor efectividad frente a las necesidades de las gestantes que experimentan VP, y las principales limitaciones para el abordaje efectivo de la VP en las gestantes que acuden a la Unidad de Servicios de Salud Usaquén. 
Las sesiones de grupos focales se realizaron en un recinto externo a la Unidad y las condujo la investigadora principal, docente experta en investigación cualitativa en género y salud, con apoyo de dos docentesinvestigadores y de estudiantes de noveno semestre de enfermería. No existía vínculo previo entre el grupo participante y la investigadora principal o el equipo de campo ni relación de sujeción alguna. Las enfermeras se dividieron en dos grupos de siete personas cada uno: los médicos y médicas conformaron un grupo y el cuarto grupo estuvo constituido por los restantes profesionales del equipo de salud. Las entrevistas tuvieron duración de una hora y media; fueron grabadas y posteriormente transcritas.

Para aportar validez al estudio se tuvieron en cuenta los criterios de triangulación propuestos por Norman K. Denzin (22). Así, se triangularon los datos obtenidos a partir de los cuatro grupos focales con diferentes grupos profesionales; se realizó la codificación por acuerdo entre investigadoras con apoyo de un software para el procesamiento de material narrativo; además, se tuvo en cuenta el criterio de confirmabilidad al presentar los hallazgos a los participantes en el estudio y recibir retroalimentación. Los datos se interpretaron a la luz de literatura científica sobre el tema.

El estudio se orientó a llevar a cabo una aproximación descriptiva de tipo exploratorio. Dado que se obtuvo un volumen de información amplio, el análisis temático se respaldó con el software ATLAS.ti 7 en las dos primeras fases del análisis: inicialmente, en la codificación abierta de la información, para construir códigos preliminares y, en un segundo momento, en la codificación axial, que dio como resultado la agrupación de códigos y construcción de categorías conceptuales. Seguidamente, cuadros descriptivos permitieron visualizar tendencias y prioridades.

En este artículo se presentan los temas relevantes que surgieron al integrar la información ofrecida por los diferentes grupos profesionales y triangular los resultados con los hallazgos de otros investigadores, por ser el propósito del estudio apoyar la posterior construcción de lineamientos generales para la acción en el nivel de atención primaria. El estudio, cuyo protocolo fue revisado por un Comité Técnico Institucional, fue considerado sin riesgo, y con base en lo expuesto en la Resolución 008430 del Ministerio de Salud (23), se utilizó consentimiento informado de tipo verbal. Se tuvo en cuenta la importancia de salvaguardar la dignidad, la integridad y los derechos de los participantes y de mantener la confidencialidad (24). Para preservar la identidad de las personas participantes, en este estudio no se utilizaron nombres propios.

\section{Resultados}

\section{Formas de violencia de pareja más frecuentes entre las usuarias}

Un primer aspecto tratado con los y las profesionales que participaron en el estudio fue cuáles son, desde su perspectiva, las formas de VP que más se destacan en el caso de las gestantes que acuden a la Unidad. Desde su visión, se presentan todos los tipos de VP, aun cuando la más frecuente es la violencia psicológica; otras formas de VP mencionadas son la económica, el abandono, la violencia sexual y la violencia física. Así lo expresa una participante del grupo de profesionales médicos:

Yo pienso que todas las formas; la física usualmente va muy ligada a la sexual, a tener relaciones sexuales con su pareja aun sabiendo que tengan alguna patología que las contraindique; la emocional también es la más frecuente, son abandonadas, son humilladas por un plato de comida que les da la familia de la pareja con la que viven.

Linares (25) considera que en la violencia doméstica con frecuencia coexisten diferentes formas de maltrato que interactúan entre sí. Las mujeres suelen ser más vulnerables ante la violencia psicológica, que es naturalizada por ellas, ya que pueden verla como una forma común de relacionarse en pareja (26). La violencia económica se correlaciona significativamente con la falta de acceso de las gestantes a servicios de salud, lo que genera un alto riesgo obstétrico. El abandono a la gestante, además de afectar la esfera emocional, 
se relaciona estrechamente con la violencia económica (27); el abandono es de una frecuencia inusitada en Colombia, donde en 2013, "se presentaron 10.902 solicitudes de dictámenes a padres que no han reconocido a sus hijos" (28).

Entre los antecedentes de las violencias que afectan a las gestantes que acuden a la Unidad de Servicios de Salud de Usaquén están, según la visión de los y las participantes, la cultura, los ciclos de violencia intrafamiliar, la disparidad en cuanto a las edades de las gestantes y de sus parejas (que en ocasiones les duplican la edad), la falta de redes de apoyo y de afecto familiar, la dependencia económica y emocional con respecto a la pareja por parte de las gestantes y el embarazo no deseado. Veamos dos de las intervenciones al respecto:

"A veces se refugian en las relaciones de pareja queriendo salir de esos contextos y lo que encuentran es que se repiten esos patrones". (Profesional del equipo de trabajo social, psicología y otros)

"Muy pocas tienen trabajo, lo que hace que dependan; que la violencia económica también sea frecuente". (Profesional de enfermería)

Según lo explican Menchón et al. (26), son diversas las razones que llevan a las mujeres a ser más vulnerables a la violencia de género; entre estas se encuentran los estereotipos, que las llevan a someterse al dominio de su pareja, la falta de recursos económicos propios, la ausencia de lazos familiares que les ofrezcan apoyo y aspectos personales, como la autoestima baja y la asunción de la violencia como forma de resolver conflictos.

\section{Estrategias propuestas para la detección de la violencia de pareja}

La exploración durante la consulta a través del diálogo asertivo resulta ser la estrategia que por excelencia se propone por parte de los participantes para la detección de la VP a la gestante, ampliando los tiempos para ello. Otras estrategias son la realización de examen físico, la aplicación de escalas de riesgo biopsicosocial, la realización de tamizajes, la implementación de protocolos de detección y el diálogo generativo de tipo grupal. También se propone la valoración interdisciplinaria en la primera consulta para realizar un abordaje integral que facilite la detección. Veamos algunas intervenciones:

"Con mucho respeto, apoyo y dignidad con la persona, tratar de llegar un poco más hacia su parte interior de alguna forma”. (Profesional médico)

"La forma de la valoración debe enfocarse mucho en la parte biopsicosocial y, de acuerdo con eso, direccionar sin demora, ni dificultades". (Profesional de enfermería)

En este estudio, tal cual lo encontró en Chile Salgado Díez (29), se revelan, en los diferentes grupos profesionales, consensos sobre la relevancia de crear espacios terapéuticos acogedores, empáticos y de contención, para el abordaje a la VP. En ocasiones, la consulta médica puede representar la única oportunidad de detener la violencia antes de que la mujer pueda sufrir lesiones de mayor gravedad (30). En este sentido, el saber cómo y cuándo realizar preguntas durante la consulta sobre VP puede hacer la diferencia (31). Se requiere que el personal de salud esté adecuadamente capacitado sobre cómo formular preguntas, a la vez que es necesario ofrecer una respuesta a las mujeres; el consentimiento de las usuarias es un aspecto crucial en este contexto, siendo de vital importancia no exponerlas ante sus parejas (32). Están más dispuestos a indagar respecto a este particular aquellos miembros del equipo de salud que se sienten más preparados sobre la problemática (33).

\section{Atención a las gestantes víctimas de violencia de la pareja}

La atención multidisciplinaria y el abordaje biopsicosocial, el fortalecimiento de la autoestima, el empoderamiento a la gestante para la comprensión de la problemática, el reconocimiento de sus derechos y redes de apoyo, el acompañamiento, la protección de su integridad y la capacitación del personal de salud para 
el abordaje la VP son las estrategias propuestas por los profesionales de salud para la apropiada atención a las gestantes que experimentan VP. Mejorar su autoestima es considerado requisito para que las gestantes sean capaces de enfrentar su realidad de manera autónoma: "Apoyar la autoestima de la gestante porque finalmente hay que empoderarlas de lo que ellas pueden hacer" (profesional médico).

$\mathrm{Al}$ respecto, Salgado afirma que trabajar con mujeres víctimas de violencia implica ayudarlas a conectar aquello que les ocurre con su socialización de género y a tomar decisiones vitales (31). El que las gestantes reconozcan sus derechos, especialmente sus derechos sexuales y reproductivos, y los defiendan es un aspecto central del proceso de atención, según lo expuesto en los grupos focales:

"Es necesario poder hacer una atención multidisciplinaria... Trabajar temas relacionados con derechos sexuales y reproductivos". (Profesional de enfermería)

"Una mujer que tenga conocimiento propio y amor propio pues siempre va a luchar porque no sean vulnerados sus derechos”. (Profesional médico)

Surgió también la importancia de que las gestantes puedan contar con acompañamiento y protección:

"Empoderar más a las mujeres en el fortalecimiento de rutas y redes de apoyo". (Profesional del equipo trabajo social, psicología y otros)

"Para no revictimizar a las usuarias entonces la idea es crear una metodología que de respuesta rápida para que ellas puedan ver que sí puede funcionar, que se sientan seguras, que tienen un apoyo”. (Profesional médico)

La capacitación al personal de salud para el adecuado manejo de la problemática fue también un aspecto importante en esta reflexión:

"La paciente lo comenta y a veces uno no sabe manejar el caso". (Profesional médico)

"A nosotras las enfermeras no nos han capacitado en intervención en crisis". (Profesional de enfermería)

La Organización Panamericana de la Salud sugiere intervenciones de profesionales especializados orientadas al empoderamiento de las mujeres y a otorgarles información suficiente sobre redes de apoyo, incluyendo el componente protección de su seguridad (32).

\section{Discusión}

Desde la perspectiva de los profesionales participantes, la cultura, en que priman las relaciones de género desiguales, aunada a falencias en la estructura intrafamiliar marcada por historias de violencia y ausencia de vínculos sólidos, constituyen el contexto donde tiene lugar la VP a las gestantes que acuden a la Unidad de Servicios de Salud Usaquén. Las gestantes suelen ser particularmente vulnerables a la VP cuando no cuentan con recursos económicos propios y redes de apoyo, lo cual es frecuente entre las más jóvenes sin acompañamiento familiar. La dependencia económica resulta ser un aspecto central de la VP, la cual se acrecienta si se entrecruza con la edad temprana. En las gestantes más jóvenes, la pobreza y la falta de educación, aunadas al escaso apoyo familiar, generan mayor vulnerabilidad (33). Además, es bien conocido que en la VP la falta de autonomía inhibe a la mujer para tomar una posición crítica y para actuar frente al maltrato que experimenta. El nivel educativo ejerce un efecto protector al vincularse con una autonomía económica y con la mayor capacidad de resolución de conflictos dentro de la relación con la pareja (34).

La violencia psicológica aparece como la más frecuente desde la visión de los participantes, lo cual coincide con los hallazgos de Baird et al., en Australia (35), quienes añaden que esta forma de violencia antecede a otras expresiones como la violencia física y sexual, siendo sus principales víctimas las gestantes de menor edad. Es de destacar que algunos estudios exponen una estrecha relación entre las bajas condiciones de vida y la presentación de problemáticas de salud mental, como la depresión gestacional en mujeres con VP (36,37). Núñez-Rivas et al. (37) plantearon la necesidad de explorar no solo las violencias física y sexual, sino la 
psicológica, así como detectar redes de apoyo psicosocial y el reconocimiento en las gestantes de problemáticas como el estrés postraumático y el desequilibrio emocional.

Los profesionales exponen que ofrecer espacios de acogida en la consulta e implementar trabajo interdisciplinario permite detectar en mayor medida la situación de las gestantes que están siendo víctimas de VP; de igual modo, alertan acerca de la importancia de fortalecer su autoestima y evitar su revictimización. Profesionales de atención primaria en salud mental, abordados por Salgado Díez (29), señalan que evitar críticas o juicios de valor son aspectos que ayudan a enfrentar casos de maltrato entre las usuarias; destacan, al igual que los participantes en este estudio, la importancia del trabajo multidisciplinario y el abordaje integral.

Partiendo de lo expuesto por los profesionales vinculados al estudio, la inclusión de una mirada de género y derechos es una necesidad a la hora de abordar la VP en los contextos de la salud, aspecto que es destacado por ONU Mujeres (2). Incrementar su conciencia en torno a los derechos que las cobijan y a los aspectos normativos implicados contribuye significativamente al cambio de la situación en gestantes con VP (38). Los derechos sexuales y reproductivos requieren estar en el centro de los procesos de empoderamiento a las mujeres, tal como lo proponen los participantes. Mientras no se garanticen la salud y los derechos sexuales y reproductivos, la igualdad de género y el empoderamiento de las mujeres y niñas no serán una realidad (39).

Es de vital importancia la preparación del equipo de salud para abordar la VP en el embarazo, según lo expuesto en los grupos focales. Es imperioso saber enfrentar esta realidad de tal manera que las mujeres salgan fortalecidas y sientan el apoyo requerido, como lo advierte la Organización Panamericana de la Salud (11). Uno de los aspectos clave está en contar con estrategias dialógicas que permitan explorar posibles violencias, que lleven a las gestantes a sentirse en confianza y a exponer libremente su situación $(31,32)$. Existe una especial preocupación de los y las profesionales por contar con recursos para enfrentar la problemática y por fortalecer su capacidad de respuesta; esta misma situación fue encontrada por otros autores como Salgado Díez (29), en Chile; Martínez Galiano (31), en España, y Arslantas et al. (38), en Turquía, lo cual lleva a pensar que se requiere definitivamente atender esta necesidad. Los profesionales de la salud demandan ser capacitados en mayor medida para hacerle frente a la VP que se presenta durante el embarazo. La formación en violencia de género por parte del personal de salud $(31,32,33,34,35)$, el conocimiento de los indicadores de sospecha, la utilización de instrumentos de detección y su decidido compromiso con respecto a la problemática y acompañamiento a las víctimas (31) son aspectos determinantes de la eficacia de los procesos. El reconocimiento por parte de los profesionales de alternativas efectivas de apoyo a las mujeres víctimas de VP es un requisito para ofrecerles orientación apropiada (32).

Autores como Ludermir et al. (36) insisten en la importancia de que las mujeres puedan contar con redes de apoyo legal, psicológico y social. Construir estrategias de intervención a favor de las mujeres que se encuentran en mayor riesgo es asunto de primer orden, dadas las implicaciones que la VP tiene para su salud mental (37). El apoyo inmediato a la gestante, a través de estrategias de información sobre opciones de soporte en casos de VP y la coordinación o derivación a otros servicios cuando sea necesario, son aspectos prioritarios en los procesos de atención (40).

\section{Conclusiones}

Desde la perspectiva de los profesionales de la salud participantes en el estudio, las relaciones asimétricas de género y la desintegración familiar, aunadas al hecho de ser jóvenes, carecer de autonomía económica y no contar con redes de apoyo, potencializan la VP a la gestante usuaria de la unidad de servicios de salud, la cual, aunque se presenta en todas las modalidades, es primordialmente de tipo psicológico. Como alternativas surgen, desde los y las participantes, propuestas para la acción que son compartidas en su mayoría por autores de otros países y continentes: inclusión de un enfoque de género y derechos, abordaje interdisciplinario, espacios de diálogo y contención, fortalecimiento de la autoestima, empoderamiento para el ejercicio de 
derechos, coordinación y derivación efectiva, minimización de la revictimización y capacitación al personal de salud para la comprensión de la violencia contra las mujeres y para la detección e intervención en VP.

Continuar trabajando para hacer de los profesionales de la salud referentes de primer orden en la violencia género ejercida por la pareja en el embarazo es un imperativo ético al que debemos responder de manera decidida.

\section{Referencias}

1. World Health Organization. Violence against women: Fact Sheet. Ginebra: WHO; 2016.

2. UN Women. Facts and figures: Ending violence against women. Fact Sheet. Washington, DC; 2015.

3. Bailey BA. Partner violence during pregnancy:prevalence, effects, screening, and management. J Womens Health. 2010;2:183-97. https://doi.org/10.2147/IJWH.S8632

4. Devries KM, Kishor S, Johnson H, Stöckl H, Bacchus LJ, García-Moreno C, Watts C. Intimate partner violence during pregnancy: analysis of prevalence data from 19 countries. Reprod Health Matters. 2010;18(36):158-70. https://doi.org/10.1016/S0968-8080(10)36533-5

5. Han A, Steward D. Maternal and fetal outcomes of intimate partner violence associated with pregnancy in the Latin American and Caribbean región. J Gynecol Obstet. 2014;124:6-11. https://doi.org/10.1016/j.ijgo.2013.06.037

6. Pallito Ch, Ocampo P. Relación entre la violencia contra la mujer en la pareja y el embarazo no planeado: análisis de una muestra nacional de Colombia. En Instituto Alan Guttmacher. Violencia basada en género y salud reproductiva. New York: Alan Guttmacher Institute; 2006. pp. 23-32.

7. Gómez A, Williams M, Sánchez S, Lam N. Intimate partner violence and risk for depression among postpartum women in Lima, Peru. Violence Vict. 2009;24(3):380-98. https://doi.org/10.1891/0886-6708.24.3.380

8. Urquía M, O’Campo P, Heaman MI, Janssen PA, Thiessen K. Experiences of violence before and during pregnancy and adverse pregnancy outcomes: an analysis of the Canadian maternity experiences survey. BMC Preg $\neg$ nancy Childbirth. 2011;11:42. https://doi.org/10.1186/1471-2393-11-42

9. Lipsky S, Holt VL, Easterling TR, Critchlow CW. Impact of police-reported intimate partner violence during preg $\neg$ nancy on birth outcomes. Interpers Violence. 2010;25(10):1928-1940. https://doi.org/10.1016/S00297844(03)00573-8

10. Organización Panamericana de la Salud. Comprender y abordar la violencia contra las mujeres: Consecuencias para la salud. Washington, DC: OPS; 2013.

11. Organización Panamericana de la Salud. Prevención de la violencia sexual y violencia infligida por la pareja contra las mujeres: Qué hacer y cómo obtener evidencias. Washington DC: WHO; 2011.

12. Colombini MS, Mayheu S, Ali SH, Shuib R, Watts CH. "I feel i is not enough..." Health providers's perspective on services for victims of intimate partner violence in Malaysia. BMC Health Serv Res. 2013;13:65. https://d oi.org/10.1186/1472-6963-13-65

13. Rees K, Zweigenthal V, Joyner K. Health sector responses to intimate partner violence:A literature review. Afr J Prim Health Care Fam Med. 2014;E:1-8. https://doi.org/10.4102/phcfm.v6i1.712

14. Hospital Usaquén ESE. Diagnóstico local con participación social 2012. Bogotá: Alcaldía Mayor de Bogotá: 2013.

15. Profamilia. 1 ${ }^{a}$ Encuesta Distrital de Demografía y Salud Bogotá 2011. Bogotá: Asociación Probienestar de la Familia Colombiana; 2012.

16. Balanta C, Viracacha A, Sanabria J. Atlas de salud pública: Localidad de Usaquén 2015. Bogotá: Alcaldía Mayor de Bogotá; 2015.

17. Lafaurie MM. Violencia de la pareja íntima en relatos de gestantes atendidas en el Hospital de Usaquén (Bogotá, Colombia). Rev Colomb Enferm. 2015;11(10):45-56. https://doi.org/10.18270/rce.v11i10.738

18. Buss Thofehrn M, López MJ, Rutz A, Coelho S, De Oliveira IC, Mikla M. Grupo focal: Una técnica de recogida de datos en investigaciones cualitativas. Index Enferm. 2013, (22):1-2. https://dx.doi.org/10.4321/S1132-129 62013000100016 
19. Hamui A, Varela M. La técnica de grupos focales. Inv Ed Med. 2013;2(5):55-60.

20. De la Cuesta C. La investigación cualitativa y el desarrollo del conocimiento en enfermería. Texto Contexto-Enferm. 2010;19(4):762-6. https://dx.doi.org/10.1590/S0104-07072010000400020

21. Martínez-Salgado C. El muestreo en investigación cualitativa: Principios básicos y algunas controversias. Ciênc Saúde Coletiva. 2012;17(3):613-9. https://dx.doi.org/10.1590/S1413-81232012000300006

22. Denzin NK. The research act: A theoretical introduction to sociological methods. New Jersey: Transaction Publishers; 1970.

23. Resolución 008430 ( 4 de octubre de 1993), por la cual se establecen las normas científicas y técnicas para la investigación en salud. Bogotá: Ministerio de salud; 1993.

24. Ley 911 (6 de octubre de 2004), por la cual se dictan disposiciones en materia de responsabilidad deontológica para el ejercicio de la profesión de enfermería. Diario Oficial 45.693.

25. Linares J. Análisis e intervención de enfermería en la violencia de género: Importancia del diagnóstico dentro del equipo de salud. Documento procedente del Tercer Congreso para el Estudio de la Violencia contra las Mujeres. Sevilla: Junta de Andalucía; 2012.

26. Menchón P (coord.), Artacho A, Castellanos P, Holgado M, Bellido J, Muñoz N. Sobre la inhibición a denunciar de las víctimas de violencia de género. Madrid: Ministerio de Sanidad, Servicios Sociales e Igualdad del Gobierno de España; 2015.

27. Sánchez N, Galván H, Reyes U, Reyes U, Reyes K. Factores asociados al maltrato durante el embarazo. Bol Clin Hosp Infant. 2013;30(1):8-15.

28. Manrique D. Pruebas de paternidad siguen rodeadas de prejuicios. UN Periódico. 2014 mayo 10.

29. Salgado Díez B. Relatos de los equipos de salud mental de atención primaria (APS) acerca del abordaje de la violencia de género en la pareja. Ciênc Saúde Coletiva. 2012;17(12):3301-10.

30. Instituto de Salud Pública. La violencia contra las mujeres considerada como problema de salud pública. Documento de apoyo a la atención en salud a las mujeres como víctimas. Madrid: Instituto de Salud Pública de la Comunidad de Madrid; 2003.

31. Martínez Galiano J. Detección y atención a la violencia de género en el embarazo: Una asignatura pendiente. Enferm Global; 2011;10(4):352-7.

32. Organización Panamericana de la Salud. Resumen: Respuesta a la violencia de pareja y a la violencia sexual contra las mujeres. Directrices de la OMS para la práctica clínica y las políticas. Washington DC: OPS; 2014.

33. Ministerio de Salud y Protección Social, Fondo de Población de las Naciones Unidas, Asociación Colom $\neg$ biana de Facultades de Enfermería. Protocolos para la atención de enfermería a la salud sexual y reproductiva de la mujer. Bogotá: Ministerio de Salud y Protección Social; 2014.

34. Cortés C I, Rivera S, Amorin de Castro E F, Rivera L. Violencia de pareja en mujeres: Prevalencia y factores asociados. Acta Investig Psicol. 2015;5(3):2224-39. https://doi.org/10.1016/S2007-4719(16)30012-6

35. Baird K, Saito A, Eustace J, Creedy D. An exploration of Australian midwives' knowledge of intimate partner violence against women during pregnancy. Women Birth. 2015;28(3):215-20. https://doi.org/10.1016/j.wom bi.2015.01.009

36. Ludermir AB, Valongueiro S, Barreto T. Common mental disorders and intimate partner violence in pregnancy. Rev Saúde Pública. 2014;48(1):29-35. https://dx.doi.org/10.1590/S0034-8910.2014048004538

37. Núñez-Rivas HP, Monge-Rojas R, Gríos-Dávila C, Elizondo-Ureña AM, Rojas-Chavarría A. La violencia física, psicológica, emocional y sexual durante el embarazo: riesgo reproductivo predictor de bajo peso al nacer en Costa Rica. Rev Panam Salud Pública. 2003;14(2):75-83.

38. Arslantas H, Adana F, Ergin F, Gey N, Biçer N, Kiransal N. Domestic violence during pregnancy in an eastern city of Turkey:a field study. J Interpers Violence. 2012;27(7):1293-313. https://doi.org/10.1177/0886260511425248

39. Federación Internacional de Planificación de la Familia (IPPF). Salud y derechos sexuales y reproductivos: La clave para alcanzar la igualdad de género y el empoderamiento de las mujeres. Londres: Federación Internacional de Planificación de la Familia; 2015. 
40. Rodríguez-Blanesa G, Vives-Casesb C, Miralles-Bueno JJ, San Sebastián M, Goicolea I. Detección de violencia de compañero íntimo en atención primaria de salud y sus factores asociados. Gac Sanit. 2017;31(5):410-5. https:/ /doi.org/10.1016/j.gaceta.2016.11.008

\section{Notas}

* Artículo de investigación

1. Financiamiento: ninguno

2. Conflictos de interés: las autoras declaran no tener conflictos de interés

Licencia Creative Commons CC BY 4.0

Cómo citar este artículo: Lafaurie Villamil MM, Ramírez Romero ML. Perspectiva de profesionales de salud sobre la violencia de la pareja en el embarazo. Investig Enferm Imagen Desarr. 2020;21(2). https://doi.org/ 10.11144/Javeriana.ie21-2.ppsv 